\title{
LA TEORÍA LITERARIA Y EL PARADIGMA: LA CIRCULACIÓN DEL INCONSCIENTE POLÍTICO
}

LITERARY THEORY AND PARADIGM: THE CIRCULATION

OF THE POLITICAL UNCONSCIOUS

Víctor Escudero

ORCID 0000-0003-2172-5812

Universidad de Barcelona, Barcelona, España

\section{Resumen:}

Desde que Fredric Jameson propuso pensar el inconsciente político de las narraciones literarias en 1981, su propuesta ha circulado frecuentemente en ámbitos ajenos a la teoría literaria (arquitectura, cine, filosofía política, etc.). Cuando el inconsciente político circula no se traspone tanto un concepto teórico de forma literal cuanto un modo de visibilidad o un paradigma que establece analogías entre ámbitos distintos incluyendo dicha diferencia como posibilidad de la circulación. A partir de la noción de paradigma de Thomas $S$. Kuhn y de la discusión propuesta por Giorgio Agamben, este texto piensa qué clase de paradigma se convoca en varios de esos usos y circulaciones, con el objetivo de discernir cómo la teoría literaria desborda sus contornos y se activa en otros discursos y disciplinas.

Palabras claves: Inconsciente político; Fredric Jameson; Circulación; Paradigma; Thomas S. Kuhn; Giorgio Agamben; Teoría literaria

\section{Abstract}

Since Fredric Jameson proposed thinking about the political unconscious of literary narratives in 1981, the concept has frequently circulated in areas outside literary theory (architecture, cinema, political philosophy, etc.). When the political unconscious circulates, it is not so much a literal concept that is transposed but a mode of visibility or a paradigm that establishes analogies between different spheres, including said difference as a possibility of circulation. Based on Thomas S. Kuhn's notion of paradigm and the discussion proposed by Giorgio Agamben, this paper scrutinizes what kind of paradigm is at stake in several of these uses and circulations,

\section{Resumo}

Desde que Fredric Jameson propôs pensar sobre o inconsciente político das narrativas literárias em 1981, sua proposta circulou frequentemente em áreas fora da teoria literária (arquitetura, cinema, filosofia política, etc.). Quando o inconsciente político circula, nem tanto um conceito teórico é transposto literalmente como um modo de visibilidade ou um paradigma que estabelece analogias entre diferentes esferas, incluindo a referida diferença como uma possibilidade de circulação. Com base na noção de paradigma de Thomas S. Kuhn e na discussáo proposta por Giorgio Agamben, este texto reflete sobre que tipo de paradigma é convocado em vários desses usos e circulaçôes, com 
with the aim of discerning how literary theory spills over its boundaries and it is activated in other discourses and disciplines.

Keywords: Political Unconscious; Fredric Jameson; Circulation; Paradigm; Thomas S. Kuhn; Giorgio Agamben; Literary Theory o objetivo de discernir como a teoria literária transborda seus contornos e é ativado em outros discursos e disciplinas.

Palavras chaves: Inconsciente político; Fredric Jameson; Circulação; Paradigma; Thomas S. Kuhn Giorgio Agamben; Teoria literária

Fredric Jameson: the purpose of theory being not to invent solutions but to produce problems in the first place.

Terry Eagleton: the ambivalence of Jameson's writing, stranded somewhere between écriture and ecrivance, can also be read as a curious doubling of commentary and critique.

En 2018, el Museo de Arte Contemporáneo de Barcelona dedicó una exposición al artista plástico Domènec en la que revisaba las utopías de la modernidad y, especialmente, sus cristalizaciones en proyectos arquitectónicos, urbanos y espaciales: desde las utopías socialistas en la línea de la Icaria de Étienne Cabet o el Pla Cerdà de Barcelona, pasando por las intervenciones arquitectónicas de Le Corbusier y otros grupos de la primera mitad del siglo XX, hasta alcanzar la última planificación urbana europea a través de los ensanches periféricos y las banlieus proyectados a partir de los años $70 \mathrm{y}$ 80. En esa exposición, tanto el propio artista (DOMĖNEC, 2019) como la comisaria (GRANDAS, 2018) y otros ensayos críticos sobre el artista (FONT i AGUSTÍ, 2002) utilizaban la noción de inconsciente político como elemento central de su proyecto estético.

El propio artista lo explica así:

He trabajado en torno a los paradigmas arquitectónicos de la modernidad, con una lectura crítica de las construcciones simbólicas de Alvar Aalto, Mies van der Rohe o Le Corbusier, en un intento de identificar la arquitectura como el «inconsciente político» de la modernidad. Como ya detectó Walter Benjamin, los proyectos de los arquitectos constituirían la mejor encarnación de todos esos sueńos de una modernidad impotente para cumplir sus promesas de emancipación y bienestar para todos. Irónicamente, las contradicciones entre el programa ideológico y la realidad política se hacen más evidentes en los proyectos de vivienda social. (2019) 
Sin embargo, en el catálogo y en los textos de la exposición no se mencionaba a Fredric Jameson, que había puesto en circulación la idea de inconsciente político en 1981 con su ensayo The Political Unconscious. Narrative as a Socially Symbolic Act en el marco de la teoría literaria. Problemas parecidos aparecen también con los usos del inconsciente político en ámbitos distantes y poco comparables (poscolonialismo, cine, democracia, etc.). Se trata de usos discontinuos, sin patrones ni una tradición común que les permita dialogar entre sí. Ni siquiera la que podría partir de una filiación confesada por la paternidad atribuida a Jameson, que a veces ni se reconoce.

Cuando el inconsciente político circula no se traspone tanto un concepto teórico de forma literal cuanto un modo de visibilidad o un paradigma que establece analogías entre ámbitos distintos incluyendo dicha diferencia como posibilidad de la circulación. Parece relevante, entonces, pensar qué clase de paradigma se convoca en esos usos y circulaciones, porque nos puede permitir discernir cómo la teoría literaria desborda sus contornos y se activa en otros discursos y disciplinas. Nos preguntaremos qué noción de paradigma está en juego, partiendo de la discusión que propone Giorgio Agamben (2010) de los usos del paradigma en La estructura de las revoluciones cientificas de Thomas S. Kuhn (1962).

\section{El código maestro del marxismo}

Jamenson, en un artículo relevante de 1971 titulado "Metacommentary", se propone la siguiente tesis: "every individual interpretation must include an interpretation of its own existence, must show its own credentials and justify itself: every commentary must be at the same time a metacommentary as well" (JAMESON, 2008, p. 7). Esa apelación a la reflexividad de la crítica que alguien que había pasado los años 50 en Francia no podía dejar de asumir, nos sirve para entender cómo la misma noción de inconsciente político apela a un código maestro (el marxismo) que la acoge en un ámbito más amplio (la lectura de la historia y las formaciones sociales) a partir de la que se puede establecer una línea de coherencia que explique, finalmente, cómo proceder al análisis literario: "in restoring to the surface of the text the repressed and buried reality of this fundamental history [the contending ruin of the contending classes]" (JAMESON, 2002, p. 4).

Ese código maestro se podría comparar a lo que Kuhn llama el paradigma sociológico en el epílogo de 1969 a La estructura de las revoluciones científicas. Kuhn escribió ese epílogo con el propósito de contener la dispersión de sus propios usos y analogías de la noción de paradigma (Margaret Masterman [1975] llega a catalogar 22 usos distintos) y agruparla alrededor de dos sentidos concretos. 
El paradigma sociológico sería una "constelación de creencias, valores, técnicas y demás, compartidos por los miembros de una comunidad dada" (2006, p. 302-303). Por lo tanto, el paradigma es asumido como universal por la comunidad científica en un momento determinado de la historia, de modo que su aceptación implica, por un lado, la construcción de un afuera (una serie de problemas dejan de ser dignos de atención), y por el otro, un desplazamiento conceptual (del geocentrismo al heliocentrismo).

Entendido de esta manera, Jameson invocaría el código maestro del marxismo como fundamentación y como estabilizador. Pero ya sabemos que no existen las invocaciones neutras: toda invocación precede a una apropiación. $Y$ algo de eso ocurre cuando el inconsciente político invoca al marxismo como fundamento y alimento. El monográfico que dedica al inconsciente político la revista Diacritics de la Universidad de Cornell $(12.3,1982)$ menos de un año después de que aparezca Political unconsciuos de Jameson es un síntoma de esa apropiación. En ese número se despliega un ejercicio de recepción crítica muy interesante, con textos y entrevistas de marxistas ilustres como Terry Eagleton, Samuel Weber o Leonard Green.

En ese debate temprano alrededor del inconsciente político no se pone tanto en entredicho como mecanismo hermenéutico, que como modo de pensar las bases marxistas desde las que el inconsciente político construye su plausibilidad. La discusión sobre el inconsciente político vehicula, por lo tanto, las tensiones internas dentro del paradigma marxista. ${ }^{1}$ A Jameson se le va a recusar, fundamentalmente en dos direcciones: la primera resulta del intento de asimilar la herencia del estructuralismo y posestructuralismo de raíz francesa, y la segunda la disolución aparentemente atrabiliaria de figuras ortodoxas y heterodoxas del marxismo (sin ir más lejos, aquellas a las que dedica su primerizo Marxism and Form en 1971: Adorno, Benjamin, Marcuse, Bloch, Lukács o Sartre; aunque sin olvidar a Williams, Althusser o Bajtin). Y ya sabemos que toda genealogía es una selección y una trinchera. Uno de los problemas que surgen, por lo tanto, alcanzada la postrimería del siglo XX, es que el paradigma marxista al que apela cada uno de ellos dista mucho de presentarse prístino y sin mácula e, incluso, dista mucho de poderse presentar como tal, de una manera completa y totalizadora.

En este sentido, resulta reveladora la propuesta de lectura de Samuel Weber. Este crítico marxista (discípulo de Paul de Man) nos invita a no desgajar el inconsciente político de cómo el marxismo en EEUU, debido a su

1 En esas discusiones, aparecen los lugares comunes de la discusión marxista a partir de lecturas más o menos fundamentalistas de los textos fundacionales: la determinación, sobredeterminación, condicionamiento entre infra y superestructura; la relación de reflejo o mediación entre una formación social e histórica determinada y la literatura; la posibilidad de invocar un discurso totalizador o utópico; la vinculación entre análisis teórico y orientación hacia la praxis transformadora; etc. (cf. EAGLETON, 1982, GREEN, et al. 1982, WEBER, 1983). 
exclusión-persecución y a la ausencia de una historia feudal como la europea, aparece como reto al establishment liberal (y no es posible encontrarlo en posiciones de prestigio, por ejemplo, académico, como en el caso de Francia o Europa). Por otro lado, Weber defiende que Jameson desafía la forma en que el postestructuralismo había sido importado a la academia americana a costa de universalizar e individualizar su obra, la cual "has thereby been purged of its conflictual and strategic elements and presented instead as a self-standind methodology" (1983, p. 16).

Weber propone acercarse al inconsciente político haciendo presente cómo la circulación de algunos corpus teóricos hacia la academia norteamericana fija las condiciones de posibilidad de lo que escribe Jameson. Y ahí ya nos vuelve a salir al paso el paradigma posestructuralista, al que Jameson reconoce el valor crítico (en la trinidad formada por Derrida, Foucault y Deleuze) aun señalando que carece de una interpretación de la historia heurística (DOWLING, 1984, p. 13). Así, si el marxismo ya empezaba a aparecerse como un paradigma problemático (si seguimos los términos de Kuhn), Jameson le incrusta el posestructuralismo, al que se podría considerar un paradigma crítico alternativo.

Convirtamos lo problemático en una oportunidad para pensar la teoría literaria. En la conocida conferencia de Bourdieu "Les conditions sociales de la circulation international des idées" (1989) ya se nos advertía que los textos circulan sin su contexto y que su importación conlleva operaciones de selección, marcaje y lectura. Teniendo esto en cuenta, en nuestro caso nos encontramos con dos problemas: el primero es que la intervención de Jameson absorbe la circulación de dos paradigmas, y el segundo radica en que la importación del posestructuralismo desafía la forma hegemónica en que dicho paradigma circula por la academia norteamericana. Ninguno de estos problemas resulta ajeno a la propia circulación de las ideas, pero nos permite detectar que, en este caso, la noción de paradigma sociológico que propone Kuhn no sirva tanto para aislar y recortar el marxismo o el posestructuralismo como para visualizar una relación paradigmática entre algunos usos de la Teoría y sus formas de pensar lo literario.

Desde esta perspectiva, la circulación del marxismo y del posestructuralismo resulta (literalmente) impensable si no se lleva a cabo a partir de núcleos condensadores, de asimilaciones acotadas o de la detección de desajustes localizados, como puede ser, entre otros, el inconsciente político como dispositivo de registro de modos y usos de la Teoría. En cierto sentido, estaría en juego una distribución de lo inteligible como la que plantea Agamben al discutir la noción de paradigma de Kuhn y confrontarla con la épistémé de Foucault y sus ejemplos paradigmáticos (el panóptico, la confesión, el cuidado de sí), que funcionan como singularidad histórica a la vez que instituyen 
inteligibilidad sobre otros fenómenos o elementos comparables (al modo de un paradigma gramatical). Agamben propone entender esos ejemplos como núcleos de inteligibilidad, que transfieren una forma de conocimiento por analogía, de lo singular a lo singular (2010: 40).

Así, por una inversión que nos conviene explorar, el inconsciente político se convertiría en la condición de posibilidad para pensar la circulación de su paradigma. De esta manera, en el ámbito humanístico, los paradigmas no serían decibles en su totalidad (a través de un manual, como ocurría en Kuhn), no serían discutibles sino a través de nociones que ayudan a cristalizarlos a la vez que los desplazan y los sintetizan. Discutamos dos ejemplos de circulación para verlo más de cerca.

\section{El inconsciente político como dispositivo ideológico}

En primer lugar, nos fijamos en el libro The Political Unconscious of Architecture. Re-opening Jameson's Narrative, editado en 2011 por Nadir Lahiji. En su texto introductorio, el editor plantea que la forma más productiva de utilizar el inconsciente político es honrándolo como condición de posibilidad de su traición (2011, p. 3): esa es la manera más adecuada de seguir su actitud crítica y contestataria contra la homogeneidad académica alrededor del liberalismo. De ahí que resalte que se apropian de la propuesta de Jameson sin transponerla ni literal ni mecánicamente a un contexto histórico y una disciplina que, forzosamente, la desplazan: "this is the letter of our return to his teaching" (2011, p. 7).

Lahiji aleja el inconsciente político de la especificidad literaria y lo traslada al análisis arquitectónico a partir de su anclaje en dos ámbitos: el marxismo y la Theory. Veamos a qué se refiere:

if it is correct to say that architecture as a social institution, and as a discourse, is also an arena in which the 'uninterrupted narrative' of the history class struggle at the level of theory is staged then it surely must be an answer to a problem that is ultimately socio-political and ethical. Zizek, in exemplary manner, links Jameson's 'political unconscious' to architecture and remarks that 'there is a coded message in an architectural formal play, and the message delivered by a building often functions as the return of the repressed of the official ideology (2011, p. 4).

Es interesante observar cómo la apropiación crítica del inconsciente político habilita a Lahiji para invocar un programa crítico de amplio alcance: reivindicación de la Theory como proyecto vigente, defensa de la conciencia ideológica y política de la labor académica, y apelación a una genealogía del marxismo que parte del Marx de la Contribución de 1859 y de Walter Benjamin. 
Lahiji y los textos que componen el volumen trasladan el inconsciente político a la arquitectura para asociarlo al análisis de obras concretas, pero también como acceso a un andamiaje teórico que lo sostiene (a la vez visible y oculto), es decir, el inconsciente político circula como gesto analítico y como dispositivo ideológico.

\section{Domènec y los paradigmas marxistas}

En el caso del artista plástico Domènec, la circulación del inconsciente político es a la vez más precisa e indirecta. Es más precisa porque parece convertirla en un concepto operativo (como llave que abre cerradura) y es más indirecta porque nunca menciona a Jameson ni a su propuesta teórica. Recordemos el planteamiento de Domènec que hemos citado al comienzo. La recusación del fracaso del proyecto emancipador de la arquitectura moderna coincide con el objetivo que también había confesado Lahiji en el texto que hemos mencionado justo antes. En las obras e instalaciones de Domènec hay una intención muy elaborada de observar cómo los procesos de construcción urbanística, funcionalización social y (posibles) reconversiones posteriores de dicha funcionalidad en los relatos oficiales, sacan a la luz las luchas y conflictos sociales que han posibilitado dichos procesos: desde la construcción de la obra pública franquista a cargo de presos políticos hasta la configuración de la marca turística de las ciudades sobre el aplanamiento de lo conflictivo, o la planificación urbanística de las periferias obreras como utopías de despacho, sin olvidar gestos más localizados como la iconoclastia y la refuncionalización de monumentos y espacios públicos.

Esta breve síntesis del discurso teórico de algunas obras de Domènec no pretende tanto sintetizar su proyecto como permitir entender cómo su apelación al inconsciente político, sin Jameson de por medio, es un mecanismo para entroncar con la relectura del materialismo histórico que Walter Benjamin propone en sus Tesis sobre filosofía de la historia (ca. 1940). ${ }^{2}$ En ningún caso se invoca al marxismo como paradigma conjunto sino a un destilado de algunas figuras de un marxismo heterodoxo que permite seguir extrayendo su potencia analítica sin confesar una adscripción sistémica. Si, por un lado, sería legítimo preguntarnos si esto es posible, es decir, si podemos extraer a la carta elementos de un código maestro como el marxismo sin perder en

2 Domènec lo explica de la siguiente manera: "trabajo con el concepto de historia establecido por Walter Benjamin, según el cual revisar la historia solo tiene sentido si es una herramienta de combate político para el presente. Me interesa estudiar qué ha pasado con estos proyectos para volver a situarlos en el presente, para que puedan ser discutidos; aportar complejidad, capas de sentido, para que juntos podamos imaginar y reimaginar alternativas" (2019). Algo parecido ocurre también con varios de los textos que interpretan a Domènec cuando remiten al inconsciente político (FONT, 2002, GRANDAS, 2018). 
esa fragmentación elemental la posibilidad de su coherencia crítica, creo que ahora nos incumbe más preguntarnos por qué ocurre.

Así, si en el uso inespecífico que hace Domènec del inconsciente político podría haber una invocación laxa al marxismo a través de Walter Benjamin, una invocación que parta de los útiles hermenéuticos del marxismo (o del materialismo histórico) pero abomine del marxismo como gran relato, entonces, ¿podríamos postular que en la circulación del inconsciente político o en sus usos analógicos estaría en juego los distintos usos del marxismo (como discurso más o menos débil, desde una posición más o menos ortodoxa) en los últimos tiempos? Si fuera así, la apelación al paradigma marxista iría acompañada de su debilitamiento como paradigma. En cierto modo, algo parecido ocurría con la asimilación de Lahiji, que utilizaba el inconsciente político como salvoconducto hacia sus filiaciones con determinadas figuras y lecturas de la Theory y del marxismo.

\section{Una reescritura de la Teoría}

La confluencia de estrategias de marcaje que hemos visto en los dos ejemplos de circulación del inconsciente político nos conduce a preguntarnos si no sería más cómodo acercar la propuesta de Jameson al paradigma ejemplar. Kuhn define este segundo tipo de paradigma como "soluciones concretas a rompecabezas que, una vez usadas como modelos o ejemplos, pueden sustituir a las reglas explícitas como base para la solución de los restantes rompecabezas de la ciencia normal" (KUHN, 2006, p. 303). De hecho, el paradigma ejemplar incluye tácitamente las leyes que rigen la investigación posterior e incorpora una promesa de éxito implícita que debe desarrollarse (KUHN, 2006, p. 89).

Al enfrentar esta versión del paradigma con los "regímenes discursivos" de Foucault con la que en ocasiones se ha podido confundir, Agamben subraya una distancia que nos puede resultar útil:

No es entonces un cambio de contenido (refutación de antiguos errores, descubrimiento de nuevas verdades), no es tampoco una alteración de la forma teórica (renovación del paradigma, modificaciones de los conjuntos sistemáticos); lo que está en cuestión es lo que gobierna [régit] los enunciados y el modo en que se gobiernan [régissent] los unos a los otros para constituir un conjunto de proposiciones científicamente aceptables y en consecuencia susceptibles de ser verificadas o invalidadas a través de procedimientos científicos (cit. en AGAMBEN, 2010, p. 17)

Mientras que el régimen discursivo es un fenómeno genuinamente político, el paradigma sería un criterio de verdad científica. En la exploración 
de esa distancia, situaremos el caso del inconsciente político. Ya hemos comprobado cómo su circulación lo transforma en gesto, salvoconducto y dispositivo ideológico. Pero recuperemos ahora su funcionamiento en Jameson como una experiencia de lectura y escritura que sirve para observar un modo de pensar la teoría literaria como un todo que solo es pensable como precondición de sentido de una de sus partes.

Al leer The Political Unconsciouss por primera vez, el lector tiene la sensación desasosegante de asistir a un patchwork de apropiaciones silvestres e insolentes de figuras y nociones sacadas de múltiples ramas y escuelas teóricas. Muchas de esas referencias son usadas puntualmente, es decir, se presentan en un apartado concreto para ponerlas en marcha en el análisis de la novela como género narrativo, o de algunas novelas secundarias de Balzac, o de Conrad o de Gissing. La única manera que el lector tiene a mano para convertir el pastiche en mosaico es acudir a los textos previos de Jameson, de los años 60 pero, especialmente, a los artículos de los años 70 (que en 1988 aparecieron recopilados en el volumen The Ideologies of Theory). Esos textos previos describen una labor de relectura consciente de la tradición crítica vigente que sitúa las bases ideológicas y los modos de apropiarse de las figuras y conceptos críticos. ${ }^{3}$

Esa condensación de toda la labor crítica previa explicaría la resistencia de The Political Unconscious, pero solo parcialmente. Lo que nos falta para acabar de entender esa resistencia tiene que ver con un solapamiento de la relectura y la escritura críticas que se produce en el proyecto de Jameson. ${ }^{4}$ Dicho solapamiento le sirve para procesar y desplazar ese bloque de la teoría francesa que ha surgido en los años 60 preguntándose por su propia discursividad (Hidalgo, 2017, p. 189), pero también para incorporar un fundamental desencaje y falta de cierre con el análisis de casos concretos, su constante hacerse cargo de nuevas referencias o del mero cambio de marco social y económico, que obliga a actualizar la posición desde la que se enuncia ("Always historicize!" es el comienzo socorrido de The Political Unconscious [2002, p. IX]).

3 A qué remite lo político en Jameson es indistinguible de su revisión de la tradición marxista en Marxist and Form (e incluso en su tesis doctoral sobre el estilo de Sartre [1961]); cómo entiende lo inconsciente parte de su relectura de Freud y, sobre todo, Lacan en "Imaginary and Simbolic in Lacan" (1977); a qué alude cuando habla de acto simbólico y narración se explica en la reescritura ideológica del proyecto de Kenneth Burke y su propuesta de la acción simbólica (1978); cómo se relaciona con la labor crítica y la hermenéutica literaria no es desgajable de su revisión de las lecturas inmanentes en The Prison-house of Language (1972) o de la tradición hermenéutica de Ricoeur o Gadamer en "Metacommentary" (1971).

4 En una línea parecida lo figura William C. Dowling en su introducción al inconsciente político: "the compression of Jameson's arguments raises the question of his own originality, which paradoxically does not lie so much in arriving at new ideas as in seeing the possibilities of synthesis in the ideas of others" (1984, p. 14). 
La lectura de Jameson suele aparecerse como una fuga en espiral en la que el método dialéctico cuestiona la posibilidad de un punto de reposo y suficiencia. Si podemos pensar, entonces, el inconsciente político a partir del paradigma ejemplar de Kuhn no es tanto en la medida en que remite a una teoría, una metodología o un hábito específicos (el marxismo? el posestructuralismo?), sino como un modo de pensar y habitar la escritura de la teoría literaria, que "está siempre afectada por la práctica o la experiencia de una lectura que no es ni simplemente una proyección de un saber previo ni el reconocimiento de una universalidad" (HIDALGO, 2020).

Entre el ejemplo y la alegoría

Cuando Agamben piensa la relación analógica entre los ejemplos paradigmáticos de Foucault o los suyos propios (el homo sacer) y aquellos fenómenos para los que construyen inteligibilidad, acude a la analogía alegórica:

Más parecido a la alegoría que a la metáfora, el paradigma es un caso singular que se aísla del contexto del que forma parte solo en la medida en que, exhibiendo su propia singularidad, vuelve inteligible un nuevo conjunto, cuya homogeneidad él mismo debe constituir. Dar un ejemplo es, entonces, un acto complejo que supone que el término que oficia de paradigma es desactivado de su uso normal no para ser desplazado a otro ámbito, sino, por el contrario, para mostrar el canon de aquel uso, que no es posible exhibir de otro modo"(2010, p. 23-24).

El primero que hace circular analógicamente el inconsciente político es el propio Jameson al ampliar sus análisis hacia manifestaciones culturales heterogéneas. Quedémonos con el cine, por ejemplo.

En The Geopolitical Aesthetic: Cinema and Space in the World System (1992), la traslación del inconsciente político no se produce de forma sistemática ni pormenorizada, sino a partir de alusiones a estrategias de análisis y ecos que invocan (pero no hacen explícita) la función de la obra de arte en las formaciones históricas. Si dejamos a un lado los comentarios microscópicos y fugaces sobre aspectos privativos de uno u otro lenguaje (la pulsión deseante de la descripción balzaquiana o la codificación de los hábitos perceptivos en los títulos de crédito de las películas), los análisis literario y cinematográfico que plantea Jameson a partir del inconsciente político confluyen en dos direcciones: la primera es la utilización de unos mismos modelos analíticos y la segunda se asocia a la relación, podríamos decir, alegórica, entre la obra de arte y la formación social que la acoge.

Sin infravalorar la especificidad de cada disciplina artística, vemos que a Jameson le interesa más ciertas nociones e impulsos transversales a algunas 
de ellas, como la de narración. En otras palabras, en la medida en que no quiere perder de vista el horizonte histórico en sus análisis inmanentes de las obras específicas, encuentra en la narración un nodo desde el que hacer pivotar dialécticamente su interés por la especificidad artística y su anclaje histórico. No se trata de la narración entendida al modo aristotélico, a la que se podría oponer un cine anti-narrativo (como el cine vanguardista o el slow cinema) o cierta novela experimental (plotless fiction). Se trata más bien de entender la narración como figuración parcial e indirecta de una verdad que, al modo benjaminiano, no se deja revelar directamente; en otras palabras, la narración es vista como la articulación de estructuras cuyas maniobras atrapan o cristalizan (más que construyen) en su decir o en su no-decir, la dialéctica de una historia conflictiva que actúa como causa ausente, es decir, solo podemos acceder a ella por su hacerse presente en las narraciones. ${ }^{5}$

Decimos hacerse presente pero más bien deberíamos decir alegorizarse:

La forma más plena de lo que Althusser llama causalidad expresiva (y de lo que él llama "historicismo") se mostrará así como una vasta alegoría interpretativa en la que una secuencia de acontecimientos o textos y artefactos históricos se reescribe en los términos de un relato profundo, subyacente y más "fundamental", de un relato maestro oculto que es la clave alegórica o el contenido figural de la primera secuencia de materiales empíricos. (GONZÁLEZ PASCUAL, 2015, p. 73)

Jameson explica cómo utiliza la alegoría en una entrevista de 1982 a propósito de la publicación de Political Unconscious: "a designation for a form able to hold radical discontinuities and incommensurabilities together without annulling precisely those 'differences'" (GREEN, 1982, p. 83). La alegoría se convierte en una noción central en los textos de Jameson a partir de entonces, pero sobre todo a partir de sus ensayos de los 80 sobre el posmodernismo (1987 y 1991). La alegoría sirve a Jameson para postular la relación dialéctica entre una concreción material y una totalidad ausente pero necesaria como precondición para construir el sentido de lo concreto e individual. ${ }^{6}$

5 Jameson toma la teoría de la historia como causa ausente de Louis Althusser para explicar la relación
causal entre base material de la sociedad y superestructura cultural. Según Althusser, no es posible pensar
esa causalidad como relación mecánica (una bola de billar que golpea a otra y la mueve), ni como
relación expresiva (cada parte expresa la esencia de un todo, a la manera del Volkgeist romántico), sino
como relación estructural: la estructura de la historia es inmanente a sus efectos o, dicho de otra manera,
"history is not a text, not a narrative, master or otherwise, but that, as an absent cause, it is inaccessible
to us except in textual form, and that our approach to it and to the Real itself necessarily passes through
its prior textualization, its narrativization in the political unconscious" (2002, p. 20). 6 Esa centralidad la lleva a aparecer en afirmaciones generales y en usos tan específicos como los de la función alegórica de los objetos tecnológicos en el cine de conspiraciones: así, los teléfonos, rastreadores, 
En este caso, Jameson toma casi de forma literal la noción benjaminiana de alegoría, tal como la reivindica en su tesis doctoral de 1925: El origen del drama barroco alemán. Allí, Benjamin aleja la noción de alegoría, en primer lugar, del tropo con que la identifica la retórica antigua (como relación mecánica entre una imagen denotativa y su significado) y, en segundo lugar, de la preponderancia del símbolo romántico (como relación orgánica entre figura y sentido). Al contrario, la alegoría para Benjamin es escritura en la medida en que queda definida por una estructura íntimamente dialéctica y el tiempo como duración y anclaje en una contingencia histórica no totalizable (2006, p. 383). Por ello, la crítica debe indicar cómo la función de la forma artística no es revelar la verdad, sino alegorizar la verdad al representarla: "convertir en contenido de verdad filosófica los contenidos fácticos históricos que se hallan a la base de toda obra significativa" (2006, p. 401). ${ }^{7}$

Creo que esta descripción de la alegoría benjaminiana describe los usos que plantea Jameson, actualizados en la imposibilidad de pensar y decir la totalidad que se vuelve ineludible en los tiempos del capitalismo tardío. Es ahí donde Jameson hace coincidir (de nuevo) su análisis de la historia y su proceder crítico: de la misma manera que el capitalismo tardío se presenta paradójicamente como capaz de absorber su propia contestación y se resiste a mostrarse completo de una vez, la crítica debe asumir la totalidad paradójicamente, a la vez como ausente y como condición de posibilidad, de la misma manera que la totalización por venir es precondición de la acción política (GREEN, 1982, p. 87). En esos fundamentos podríamos encontrar el inconsciente político de Jameson como crítico.

Tal vez sea eso lo que le hace mostrarse siempre reacio a adscribirse a un solo paradigma crítico, pero a la vez, intente siempre una praxis crítica que trata de agotar la posibilidad de una síntesis global de la teoría. Desde sus primeros textos a sus últimas entrevistas, Jameson siempre ha rehuido adscribirse a alguna de las escuelas que convocan las figuras y referencias que hemos visto que sus textos utilizan. Así, siempre que puede, Jameson plantea huir del uso dogmático de las escuelas o los críticos como religiones, de los

cámaras de vigilancia, procesadores de información, descodificadores de mensajes, fotocopiadoras, etc. que protagonizan la primera secuencia de The three days of Condor (POLLACK, 1977) "when grasped as the allegories of something else, of the whole unimaginable decentered global network itself" (1995, p. 13).

7 En esa evolución, Benjamin observa cómo la asociación alegórica uno a uno de un elemento representado con un significado determinado, empieza a complicarse con la aparición de combinaciones, composiciones y reelaboraciones de los mismos signos en distintas culturas. Así, la relación inicial, básica y unívoca, guiada por una cierta analogía, pierde progresivamente ese sustento, y se convierte en una asociación arbitraria, en una forma de escritura. Sin una verdad a priori que determine y garantice la relación entre A y B, las nuevas prácticas alegóricas se distancian cualitativamente de la formulación medieval, aun partiendo de ella. Para Benjamin, la alegoría deja de ser una asociación irreversible y se convierte en el proceso mismo de significación. Y así es como alegoría y escritura terminan por coincidir. 
grupos que se apropian de un crítico concreto o de las ideologías puras. ${ }^{8}$ Como hemos comentado, Jameson prefiere poder abrazar a diestro y siniestro a partir de la desestabilización que permite la dialéctica. En definitiva, Jameson es un ecléctico. Él mismo lo asume y exhibe (GREEN, 1982, p. 87). ${ }^{9}$

¿Cómo puede sobrevivir un ecléctico apelando a discursos tan autocentrados y aglutinadores como el marxismo o el estructuralismo? Pensando la síntesis de la Teoría como posibilidad utópica. En cada momento de su trayectoria, Jameson parece pretender una inquisición dialéctica de los paradigmas críticos del momento en un discurso que parte de la posibilidad de la unidad no como síntesis reparadora, sino como antagonismo: "the purpose of theory being not to invent solutions but to produce problems in the first place" (JAMESON, 2013, p. 191). Y en ese proyecto es tan importante lo tentativo y fragmentario (en la estela de la interrogación al conocimiento, y no a la verdad, de Benjamin) como la necesidad de postular una comunidad de propósito. La totalidad postulada, negada o sobreseída aparece siempre como el lugar donde se expone la ideología de la crítica.

En Jameson convergen dos labores de reescritura ideológica que son una sola: por un lado, "the rewriting of the literary text in such a way that it may itself be grasped as the rewriting or restructuration of a prior ideological or historical subtext" (2008, p. 148) y, por el otro, lo que él llama el discurso del analista, capaz de ser presente y negarse para poder articular, desatar y desmistificar las corrientes profundas del deseo colectivo (2008, p. 117):

una lectura que opera detectando los restos (las "ruinas", diría Benjamin) fragmentarios y dispersos del inconsciente político, no para reconstruir "arqueológicamente" el "edificio" original, sino para construir uno nuevo. Es un modo de interpretación crítica y sintomática cuya finalidad no es la

8 Un ejemplo entre muchos: "insist on either of these two dimensions of symbolic action without the other-to overemphasize the way in which the text organizes its subtext (in order, presumably, to reach the triumphant conclusion that the "referent" does not exist) or on the other hand to stress the instrumental nature of the symbolic act to the point where reality, understood no longer as a subtext but rather as some mere inert given, is once again delivered over into the hands of that untrustworthy auxiliary, Common Sense- to stress either of these functions of symbolic action at the expense of the other is surely to produce sheer ideology, whether it be, in the first alternative, the ideology of structuralism or, in the second, that of vulgar materialism" (2008, p. 149).

9 Su eclecticismo dialéctico parte de una conciencia política de la tarea crítica y de un rechazo de aquel discurso académico que incorporan la lógica del capital en la reflexión crítica: "What bothers me, when we have to do with the Marxist tradition, is the transformation of these various thinkers (and not only Althusser or Lukacs, but also: Gramsci, Benjamin, Brecht, Williams, Thompson, etc) into brand-names for autonomous philosophical systems. In this the Marxist tradition has itself been infected by the logic of commodity culture in general: where the organization of consumption around brand-names determines a quasi-religious conversion, first to the great modern artists (you convert to Proust, or to D. H. Lawrence, or to Faulkner, or to Sartre, etc) all incompatible, but every so often one switches religions (and then in a later stage to the theorists, so that one now converts to Heidegger, or Ricoeur, or Derrida, or Wayne Booth, or Gadamer, or De Man)" (GREEN, 1982, p. 78). 
develación de un sentido pretendidamente originario, sino la producción de un sentido nuevo, incluso la producción de silencios o vacios en el texto, que el texto ni siquiera "sabía" que existían. Y la producción de sentido es, a su vez y en sí misma, política, en tanto siempre replantea, en su propio terreno, la relación de los sujetos con los discursos de la polis. Y esto hace, entonces, que Jameson establezca una prioridad de la interpretación política de los textos y discursos culturales. (GRÜNER, 2015, p. 7)

Esa forma de pensar lo literario sería una causa ausente que cristaliza en el inconsciente político como reescritura de la Teoría e inscripción en la teoría: "el fenómeno, expuesto en el medio de su cognoscibilidad, muestra la totalidad de la cual es paradigma” (AGAMBEN 2010, p. 36).

Coda: un paradigma singular

Cuando Lahiri invocaba el inconsciente político como dispositivo de análisis en el ámbito de la arquitectura asumía la imposibilidad de su trasposición literal, pero absorbía la alegoría de una forma de practicar y poner en movimiento la Theory. Con una filiación algo distinta, Domènec reclamaba el inconsciente político como gesto de actualización del materialismo histórico. En ambos casos, la circulación del inconsciente político cristaliza como singularidad que hace visible y pensable un espacio de problemas y una forma de acceder al mismo. Por lo tanto, la exhibición de la singularidad del inconsciente político no sirve tanto para replicar su literalidad en otros ámbitos cuanto "para mostrar el canon de aquel uso, que no es posible exhibir de otro modo" (AGAMBEN, 2010, p. 24). Así es como lo singular se convierte en paradigmático. ${ }^{10}$

La circulación de un paradigma en el ámbito de la teoría literaria (y, tal vez, en el más amplio de las ciencias humanas) no puede pensarse como trasposición o sustitución, como proponía Kuhn para las ciencias naturales. La circulación funciona más bien a partir de analogías singulares o de lo que Masterman llamaba "concordancia inexacta" (1975), es decir, la imposibilidad de encontrar con exactitud en dos singularidades históricas diferentes los patrones fundamentales que singularizan a ambas. Es esa concordancia inexacta la que el propio Jameson moviliza para usar el inconsciente político en otros ámbitos y lenguajes (como el cine). De ahí que la propuesta de Jameson no funcione ni sea óptima como método, herramienta o teoría coherente y autosuficiente sino como una tarea de fijación y renuncia, un desplegarse ramificado e inacabado, esto es, como un modo paradigmático de pensar la

10 Algo parecido a lo que ocurre cuando pensamos un paradigma gramatical (un ejemplo de declinación, un modelo verbal): la relación entre los términos remite a la equivalencia sin identidad. 
teoría literaria y, en sus usos en otros ámbitos, un modo de pensar desde la exterioridad de la teoría literaria.

\section{Referencias}

AGAMBEN, Giorgio. ¿Qué es un paradigma?. In: Sobre el método. Barcelona: Anagrama, 2010: 11-42.

BENJAMIN, Walter. El origen del Trauerspiel. In: Obras 1. Madrid: Abada, 2006.

BENJAMIN, Walter. Sobre el concepto de historia. In: Obras 1/2. Madrid: Abada, 2006.

DOWLING, William C. Jameson, Althusser, Marx. Cornell University Press, 1984.

EAGLETON, Tery. The Politics of Styl”. Diacritics, v. 12, n. 3, 1982.

FONT, Jordi. Huellas del futuro, ruinas del pasado. In: Existenzminimum. Girona: Espais d'Art Contemporani, 2002.

GONZÁLEZ PASCUAL, Alberto. Análisis crítico del pensamiento político de Fredric Jameson. Sevilla: Universidad Pablo Olavide, 2015.

GRANDAS, Teresa. Domènec. Ni aquí ni enlloc. In: Ni aqui ni enlloc. Macba: Barcelona, 2018.

GREEN, Leonard et al. Interview: Fredric Jameson. Diacritics, 12.3, 1982.

GRUNER, Eduardo. Palabras que matan. Topia, 75, 2015.

HERRERA, María Victoria T. Entrevista con Domènec. Perro berde, 2019. http:// www.perroberde.com/article/entrevista-con-domenec

HIDALGO, Max. La Teoría de la Literatura: pasado y presente de un problema. Badebec, v. 7, n. 13, 2017.

HIDALGO, Max. Modelos y problemas en el estudio de la circulación de la teoría literaria: Pierre Bourdieu (1989), Pascale Casanova (1999) y el secuestro del Barroco”. Alea, v, 22, n. 3, p. 17-42, 2020.

JAMESON, Fredric. The Geopolitical Aesthetic: Cinema and Space in the World System. Indiana University Press, 1995.

JAMESON, Fredric. The Political Unconscious. Londres: Routledge, 2002.

JAMESON, Fredric. The Ideologies of Theory. Londres: Verso, 2008. 
JAMESON, Fredric. The Antinomies of Realism. Londres: Verso, 2013.

KUHN, Thomas S. La estructura de las revoluciones cientificas. México DF: FCE, 2006.

LAHIJI, Nadir (ed.). The Political Unconscious of Architecture. Re-opening Jameson's Narrative. Londres: Routledge, 2011.

WEBER, Samuel. Capitalizing History: Notes on the Political Unconscious. Diacritics, v.13, n.2, 1983.

Víctor Escudero Prieto. Profesor Serra-Hunter de la sección de Teoría de la Literatura y Literatura Comparada de la Universidad de Barcelona (UB) e investigador en el Grupo sobre Literatura, Cine y otros Lenguajes Artísticos (GLiCiArt). Ha realizado estancias de investigación en la Universidad de Buenos Aires, University of Cambridge y Harvard University. Sus principales campos de investigación son las migraciones de la teoría literaria en el cine y la esfera audiovisual, y las variaciones de la novela de formación como subgénero novelístico.

E-mail: vescudero@ub.edu 